\title{
Managing the energy trilemma in the Philippines
}

\author{
Josef T. Yap ${ }^{1 *} \mathbb{D}$, Aaron Joseph P. Gabriola² and Chrysogonus F. Herrera ${ }^{2}$
}

\begin{abstract}
Background: The transition to an energy mix with lower carbon emissions is hampered by the existence of the so-called Energy Trilemma. The primary consequence is a trade-off between various objectives of energy policy, e.g., equity and sustainability. This conflict can lead to policy gridlock if policymakers are unable to prioritize the goals. This paper proposes a framework and methodology to manage the trilemma by applying methods related to multi-criteria decision-making in order to assign weights to the various components of the trilemma.

Results: Following the International Energy Agency (IEA), an expanded concept of energy security is adopted and translates to a version of the trilemma different from that of the World Energy Council. This study takes into account autarky, price, supply, and carbon emissions. The values of these variables are generated by a software called PLEXOS and are incorporated in a welfare function. Trade-offs and complementarities among the four variables are taken into account by the equations in the PLEXOS model. Meanwhile, weights for each of the components of the trilemma are obtained using the Analytical Hierarchy Process. The experts interviewed for this exercise are considered hypothetical heads of the Philippine Department of Energy (DOE).

Conclusion: Two scenarios were compared: a market-based simulation and one where a carbon-tax was imposed. As expected, the carbon-tax leads to a fall in the level of carbon emissions but a rise in the cost of electricity. Because the demand for electricity has a higher price elasticity among lower income classes, the carbon-tax will worsen equity. Attempting to resolve the conflict among the goals of energy policy is difficult leading to a possible gridlock. Policy options can, however, be ranked using the values generated by the welfare function. The ranking clearly depends on the preference or priorities of the hypothetical head of the DOE but at least a decision could be reached. In this manner, trade-offs are measured and the trilemma can be managed even if it is not resolved.
\end{abstract}

Keywords: Energy trilemma, Energy security equity and sustainability, Policy gridlock, Multi-criteria decision-making, Welfare function

\section{Background}

Energy poverty continues to be a major concern in the Philippines, especially when compared with its neighbors in Asia. One aspect of energy poverty is household access to electricity. Table 1 shows that as of 2018, the Philippines has the lowest electrification rate among Asian countries with a similar level of development. Meanwhile,

\footnotetext{
*Correspondence: josef.t.yap@gmail.com; asep-cellspmo@ateneo.edu

${ }^{1}$ Ateneo School of Government, Quezon City, Philippines

Full list of author information is available at the end of the article
}

Table 2 shows that in 2020 the Philippines had the lowest per capita consumption of electricity in the same set of countries. It is not a coincidence that the Philippines also has one of the lowest levels of development as measured by per capita gross domestic product (GDP).

To address the problem of energy poverty, the Philippine Department of Energy targeted 100 percent electrification of households with access to the grid by 2022 . For off-grid areas, the 100 percent electrification rate is expected by 2040 . The objective dovetails with one of the major components of Sustainable original author(s) and the source, provide a link to the Creative Commons licence, and indicate if changes were made. The images or other third party material in this article are included in the article's Creative Commons licence, unless indicated otherwise in a credit line to the material. If material is not included in the article's Creative Commons licence and your intended use is not permitted by statutory regulation or exceeds the permitted use, you will need to obtain permission directly from the copyright holder. To view a copy of this licence, visit http://creativecommons.org/licenses/by/4.0/. The Creative Commons Public Domain Dedication waiver (http://creativeco mmons.org/publicdomain/zero/1.0/) applies to the data made available in this article, unless otherwise stated in a credit line to the data. 
Table 1 Electrification rate (\% of population) for selected Asian countries

\begin{tabular}{lllllll}
\hline & $\mathbf{1 9 9 0}$ & $\mathbf{1 9 9 5}$ & $\mathbf{2 0 0 0}$ & $\mathbf{2 0 0 5}$ & $\mathbf{2 0 1 0}$ & $\mathbf{2 0 1 8}$ \\
\hline Indonesia & 61.7 & 66.9 & 86.3 & 86.2 & 94.1 & 98.5 \\
Malaysia & 93.9 & 95.6 & 97 & 78.6 & 84 & 100 \\
Philippines & 62.1 & 67.9 & 83.5 & 92.3 & 99.7 & 94.8 \\
Thailand & 75.9 & 81.7 & 86.2 & 96.1 & 97.6 & 100 \\
Viet Nam & 74.1 & 80.3 & &
\end{tabular}

Source: World Bank [1]

Table 2 Per capita electricity consumption and per capita GDP in selected Asian countries

\begin{tabular}{lcc}
\hline & $\begin{array}{l}\text { Per capita electric power } \\
\text { consumption, kWh, 2020 }\end{array}$ & $\begin{array}{l}\text { Per capita GDP (at } \\
\text { constant 2010 USD), } \\
\mathbf{2 0 2 0}\end{array}$ \\
\hline China & 3991 & 8405 \\
Indonesia & 799 & 4312 \\
Malaysia & 4193 & 11,637 \\
Philippines & 717 & 2980 \\
Singapore & 7680 & 56,349 \\
Thailand & 2736 & 6094 \\
Viet Nam & 1451 & 2133 \\
\hline
\end{tabular}

Source: electricity consumption: [2]; GDP: World Bank [3]

Development Goal (SDG) 7 which is to ensure universal access to affordable, reliable, sustainable, and modern energy by 2030. However, SDG 7 also targets a substantial increase in the share of renewable energy in the global energy mix. Hence, the increase in access must be accompanied by a transition from fossil fuels to renewable energy.

Achieving increased access and a higher share of renewable energy requires managing the so-called Energy Trilemma. This refers to "the conflicting goals that governments face in securing energy supplies, providing universal energy access, and promoting environmental protection" (World Energy Council [4]). The Energy Trilemma is defined across three dimensions (Fig. 1). "Energy Security reflects a nation's capacity to meet current and future energy demand reliably and withstand and bounce back swiftly from system shocks with minimal disruption to supplies. Energy Equity assesses a country's ability to provide universal access to affordable, fairly priced, and abundant energy for domestic and commercial use. Environmental Sustainability of Energy Systems represents the transition of a

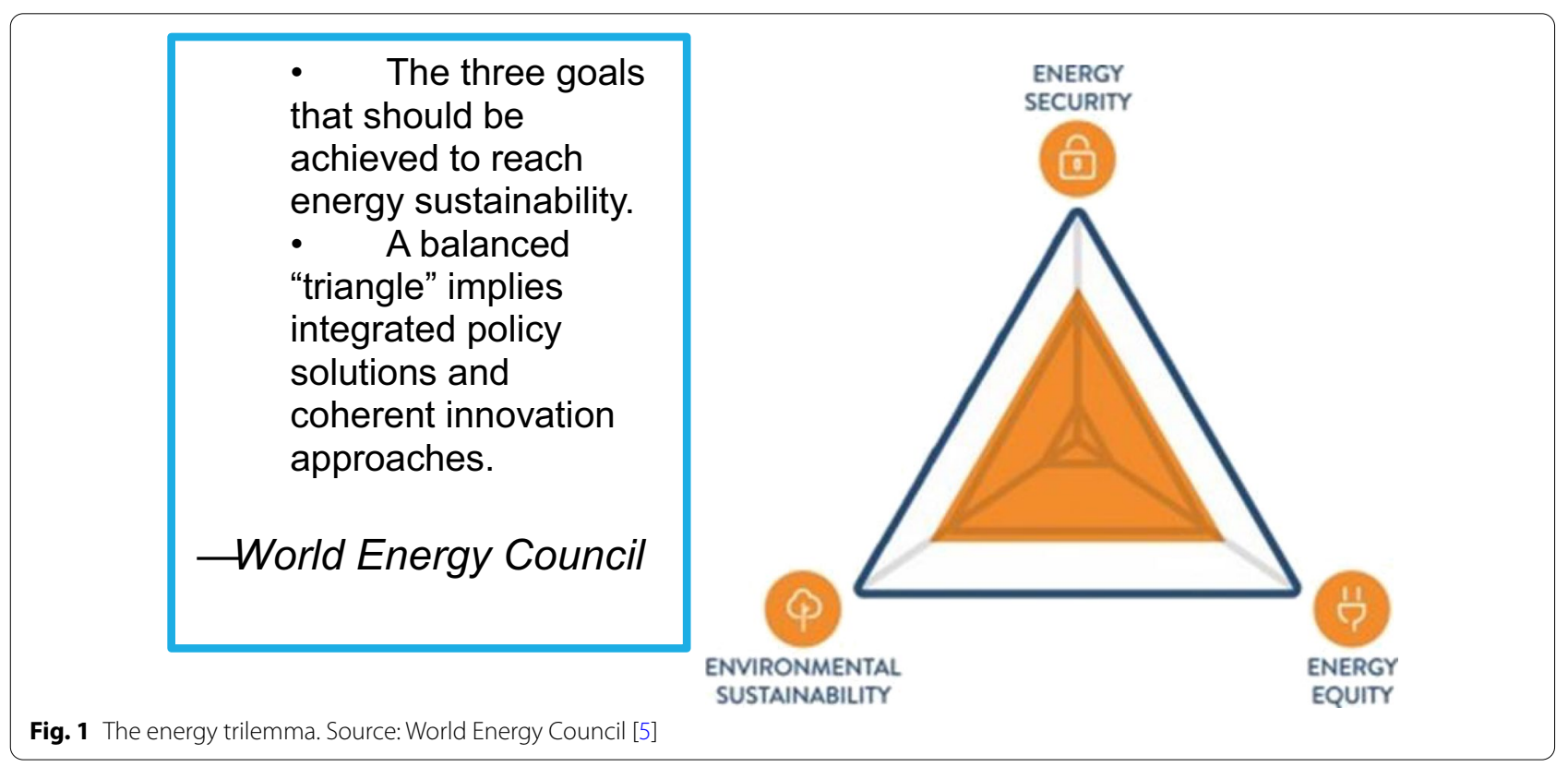


Table 3 Summary of mean levelized cost of energy (LCOE) for different energy sources

\begin{tabular}{|c|c|c|c|c|}
\hline & Fuel source & $\begin{array}{l}\text { 2009-USD/ } \\
\text { MWh }\end{array}$ & $\begin{array}{l}\text { 2018-USD/ } \\
\text { MWh }\end{array}$ & $\begin{array}{l}\text { 2019- } \\
\text { USD/ } \\
\text { MWh }\end{array}$ \\
\hline 1 & Wind & 135 & 42 & 41 \\
\hline 2 & Solar & 359 & 43 & 37 \\
\hline 3 & $\begin{array}{l}\text { Combined cycle } \\
\text { gas turbine }\end{array}$ & 83 & 58 & 56 \\
\hline 4 & Coal & 111 & 102 & 109 \\
\hline 5 & Nuclear & 123 & 151 & 155 \\
\hline 6 & Geothermal & 76 & 91 & 91 \\
\hline 7 & Hydropower & $40^{a}$ & $52^{b}$ & 54 \\
\hline 8 & Biomass & 89 & $108^{c}$ & 100 \\
\hline
\end{tabular}

Source of data: Lazard [7]

a 2010 LCOE for hydropower from the International Renewable Energy Agency (IRENA) Database. ${ }^{\mathrm{b}, \mathrm{c}} 2018$ and 2019 LCOE data collected from the Annual Technology Baseline Website of the National Renewable Energy Laboratory

country's energy system toward mitigating and avoiding potential environmental harm and climate change impacts."

Typically, the topic of sustainability should cover the concept of the environomy, which is the union of the environment and the economy (e.g., Ravago and Roumasset [6]). This would then involve a broader trilemma involving prosperity, equity and environmental sustainability. The Energy Trilemma has a narrower focus.

Resolving the Energy Trilemma entails designing policies wherein trade-offs among goals can be avoided. This is a highly unlikely scenario and in the event of relatively large trade-offs, a policy gridlock may ensue. The key research question addressed in this paper is how to move past this possible gridlock. Instead of attempting to resolve the Energy Trilemma, a framework is developed to manage it by quantifying the trade-offs among the conflicting goals. Weights reflecting the preferences of policymakers are assigned to these goals thereby prioritizing them. Policies can then be ranked through a welfare function that combines quantitative measures of the different goals. Even if conflicts among the goals cannot be resolved, progress can be made by adopting policies that have a higher welfare rank.

\section{Situating the research}

\section{Trade-offs and synergies}

The term "trilemma" implies that trade-offs are involved when energy policies are designed and implemented. For example, ten years ago, significantly increasing the share

\footnotetext{
${ }^{1}$ World Energy Council [5], page 13.
}

of variable renewable energy (VRE) like solar would have been infeasible because of the prohibitive costs involved (Table 3). The trade-off between equity, particularly affordability, and sustainability was quite clear-cut. Nowadays, because of the sharp decline in the cost of solar power generation, the trade-off emanates from the feasibility of integrating VRE in the grid system. In this context, the high cost of battery storage is the major factor that prevents the full utilization of wind and solar power in the grid system.

Thus, despite the sharp decline in generation costs involving VRE, the energy trilemma remains a problem that has to be managed. This paper proposes a methodology to achieve this objective. The approach is inspired by Barbier and Burgess [8] who evaluate trade-offs and complementarities-or synergies-among the SDGs. They adopt accepted methods to calculate changes in welfare under specified constraints. This allows measuring welfare effects of an increase in the level of one SDG while taking into account trade-offs or complementarities with other SDGs. In their study, a quantitative evaluation of progress over 2000-2016 for each of the 17 SDGs is carried out using a representative indicator for each goal. Their results have important implications for policies designed to achieve the SDGs. In particular, because synergies are taken into account, policies can be calibrated to be consistent with the priorities of policymakers.

The essence of the framework in this study is specifying a welfare function $W$ that is dependent on the components of the trilemma. One such specification is as follows:

$$
W=\text { Security }^{\alpha} \text { Equity }^{\beta} \text { Sustainability }^{\gamma}
$$

Different policies will yield different values for the three components of the trilemma, i.e., security, equity, and sustainability, thereby generating a set of values for $W$. This will enable policymakers to rank the policies. A conventional simulation package can generate the values of the three components, taking into account the tradeoffs and complementarities among them. The obvious challenge is to arrive at reasonable values for the parameters $\alpha, \beta$, and $\Upsilon$. They represent the preferences of the policymakers, which in turn, should ideally reflect the aspirations of society. Methods under multi-criteria decision-making (MCDM) can be applied for this purpose.

Being able to rank policies will facilitate decision-making. Progress can therefore be achieved even if the conflicts or trade-offs cannot be resolved. This is the essence of managing the trilemma. The choice of the term "manage" is deliberate as "resolving" the trilemma is a difficult task. 


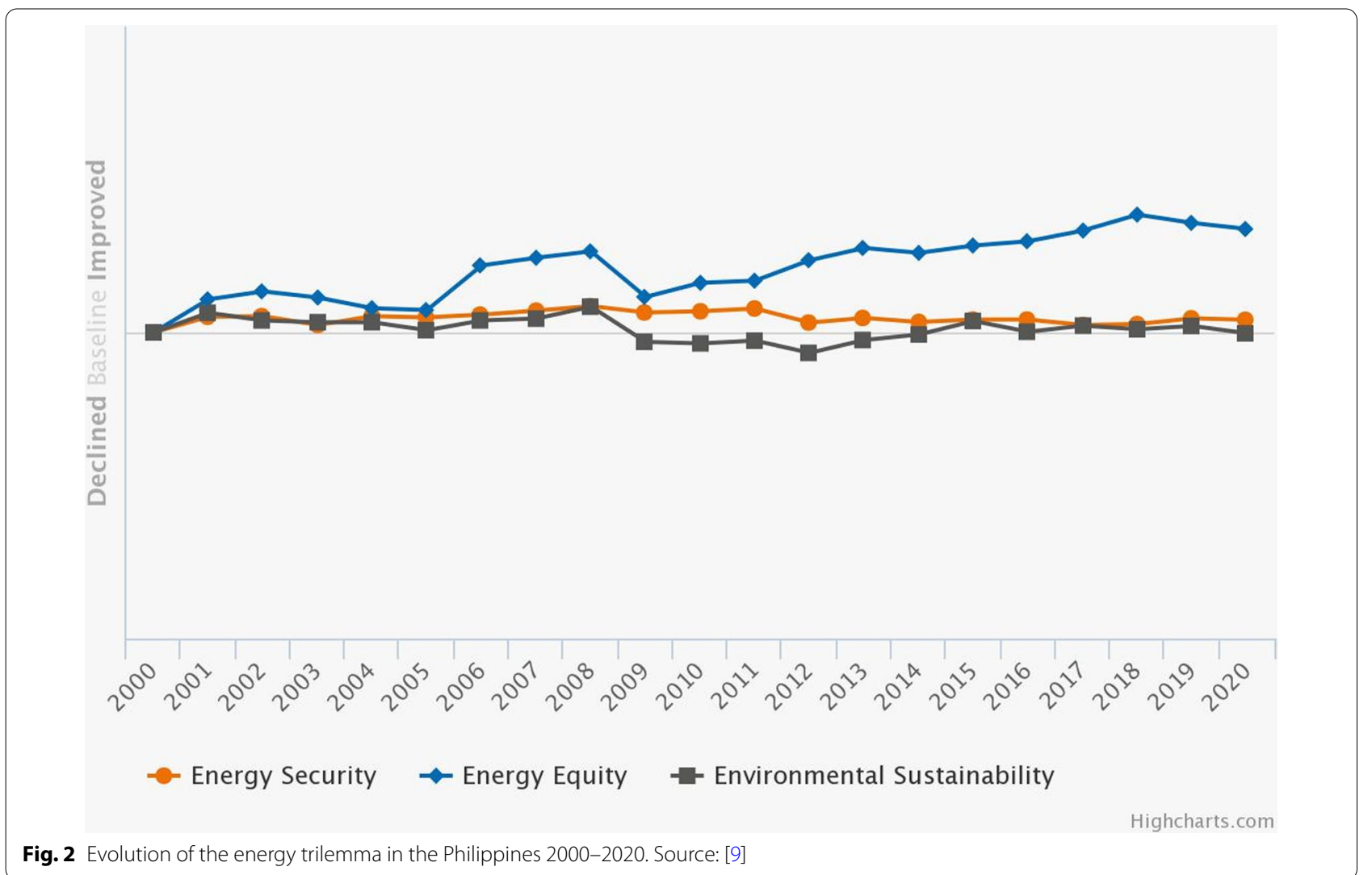

\section{Review of literature}

Energy trilemma is recognized as a global challenge. To track progress in coping with this challenge, the World Energy Trilemma Index has been prepared annually since 2010 by the World Energy Council. In its latest publication, WEC [9] presents a comparative ranking of the energy systems of 108 countries. An assessment of a country's energy system performance is also provided, based on the balance and progress in the three components of the Trilemma. The performance of the Philippines is shown in Fig. 2. The country is ranked 76th in terms of balance and progress in the different components of the trilemma.

The literature identifies strong and weaker versions of the trilemma. The former calls for policymakers to choose two of the three policy goals. This implies that the trilemma cannot be resolved but only managed. On the other hand, the weaker version recognizes that political, economic, and institutional reforms can lead to progress in all three components. Hence, from this perspective, the trilemma can be resolved by overcoming structural barriers through appropriate policy measures.

Examples of studies that adopt the weaker version of the trilemma are country cases for the Philippines (La Viña et al. [10]) and Indonesia (Gunningham [11]). The discussion largely revolves around policies that govern the transition into a greater share of low-carbon sources in the energy mix. In the case of the Philippines, the authors argue that policymakers can and should work at two categories of reform: rationalization and diversification.

At the core of rationalization efforts is a long-term energy plan that is impervious to shifts in government administrations. If this plan is perceived as robust, it will reduce political and regulatory risk, and at the same time encourage investments in the energy sector that will promote the goals of energy security, equity, and sustainability. Such a plan should also be cognizant of global technological developments which will discourage unnecessary subsidies for specific energy sources. Government-private sector coordination and publicprivate partnerships can be supported by a program such as the Competitive Renewable Energy Zones or CREZ (Lee et al. [12]). This is an example of an energy mapping system that identifies optimal areas for development vis-à-vis available energy sources and transmission lines. Overall, rationalization entails less emphasis on liberalization-or a market-led approach-and a greater role for government regulation. 
Meanwhile, the thrust of diversification is reducing the country's relatively heavy dependence on fossil fuel, particularly imported coal. The main obstacle to attaining this objective is the limited ability of renewable energy to perform the role of coal power plants as a source of baseload capacity. At present, the Philippines has an excess supply of coal plants that exceeds baseload needs, making it necessary for these coal plants to provide the mid-merit requirement. Policies have to be enacted to allow sources that can support the mid-merit requirement more efficiently than coal. "To address this, a cap on approved coal endorsements using a portfolio-based regional energy plan detailing the baseload, mid-merit, and peaking requirements in each of Luzon, Visayas, and Mindanao is necessary. This prevents an oversupply of coal plants beyond baseload needs, and, for the longterm, contractual lock-in of coal supply beyond what is economically, socially, and environmentally acceptable." ${ }^{2}$

Indonesia is a resource-rich country that plays a significant role in the global energy market. However, its per capita consumption of electricity is relatively low (Table 2). One reason for this is a strategy that encourages exports of energy resources and heavy dependence on coal. Gunningham [11] recommends effective energy governance to increase access, reduce fuel subsidies, and at the same time, facilitate the transition of the energy sector to one with lower carbon emissions. Four important elements of the governance structure have to be analyzed.

First, there is a need to instill norms-or standards of appropriate behavior-related to the importance of climate change. International organizations like the International Energy Agency (IEA) have an important role to play in convincing Indonesian policymakers of the importance of measures related to climate change adaptation and mitigation. Second, many stakeholders including international and local NGOs have argued against the implementation of fuel subsidies. ${ }^{3}$ Third, global energy governance can also help address the biggest challenge to Indonesia's transition to a low-carbon scenario: the lack of financial resources that can underwrite a revolution in the energy sector. The more prominent financing tools include the Global Environment Fund (GEF) and the climate change funds of the World Bank, most notably the Clean Technology Fund. Neither of these initiatives has offered the financial resources needed to overcome Indonesia's climate change challenges. "If such carrots

\footnotetext{
2 [10], page 43.

3 The paper of Gunningham was published in 2013. The Indonesian government eliminated gasoline subsidies in 2015 and set fixed subsidies for diesel. For more details, please refer to https://www.oecd.org/fossil-fuels/ publication/Indonesia\%20G20\%20Self-Report\%20IFFS.pdf.
}

do not achieve the necessary changes (and they are small compared to the current cost of energy subsidies to the Indonesian budget of some $\$ 20$ billion per annum), there remains the possibility of the use of sticks. Of the latter, the most plausible are carbon border taxes: taxing goods from countries that do not commit to climate change mitigation in order to ensure that those who do are not disadvantaged."

The preceding discussion highlights the difficulty of designing policy to resolve the energy trilemma. Moreover, the policies will still likely involve trade-offs. Managing the trilemma can be facilitated if the trade-offs can be quantified. A straightforward approach is the adoption of portfolio-based techniques widely used in financial markets. The general objective is to balance short-term costs with medium- to long-term price stability. The standard methodology is Markowitz's mean-variance analysis to determine the optimal energy mix for electricity generation.

A recent application is the case of the Philippines (Balanquit and Daway-Ducanes [13]). In their study, they consider eight generating technologies, each associated with two important parameters: the expected rate of return $r_{\mathrm{i}}$ and the risk measured by the variance in the return. These parameters are both derived from the technology's daily power price (PP) ratio, defined as the amount of energy sold or discharged over its average price.

$$
\begin{aligned}
& r_{i}=E\left[\frac{\mathrm{PP}_{i t}-\mathrm{PP}_{i(t-1)}}{\mathrm{PP}_{i(t-1)}}\right], \\
& \sigma_{i}^{2}=E\left[\left(\frac{\mathrm{PP}_{i t}-\mathrm{PP}_{i(t-1)}}{\mathrm{PP}_{i(t-1)}}\right)^{2}\right]-r_{i}^{2}, \\
& E(r)=\sum_{i=1}^{8} \alpha_{i} r_{i},
\end{aligned}
$$

where $\alpha_{i} \in(0,1)$ is the share of technology $i$ and that $\sum_{i} \alpha_{i}=1$.

On the other hand, the expected portfolio risk is given by

$$
\operatorname{Var}(r)=\sum_{\mathrm{i}=1}^{8} \alpha_{\mathrm{i}} \sigma_{\mathrm{i}}^{2}+2 \sum_{1 \leq \mathrm{i} \leq \mathrm{j} \leq 8} \alpha_{\mathrm{i}} \alpha_{\mathrm{j}} \sigma_{\mathrm{ij}},
$$

where $\sigma_{i j}$ is the covariance of two distinct technologies $i$ and $j$. The methodology then adopts the approach of Markowitz [14] by minimizing a given portfolio's risk

\footnotetext{
${ }^{4}$ [11], pages 190-191.
} 


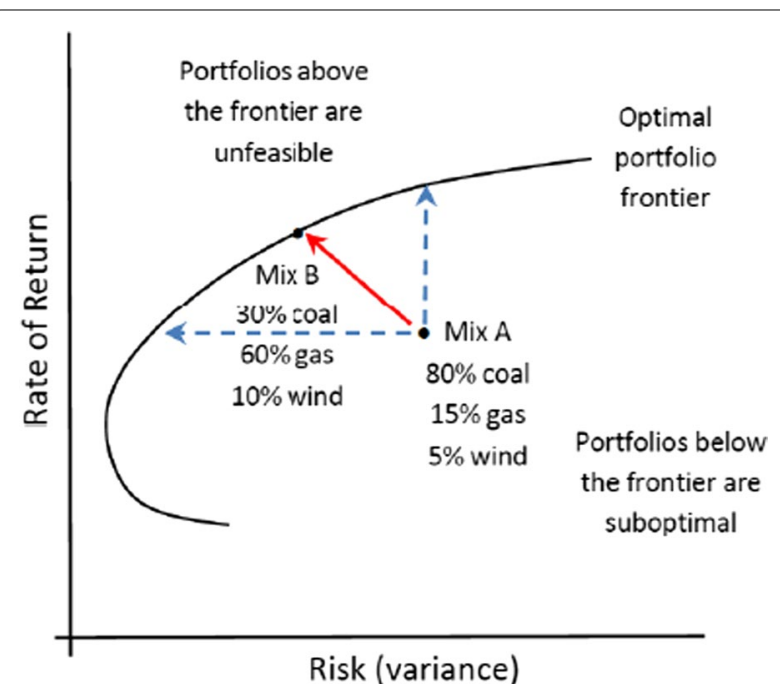

Fig. 3 An example of an optimal portfolio frontier. Source: Fig. 6.1 of [13]

for every targeted rate of return $r$. The problem can be depicted as:

$$
\begin{aligned}
& \min _{\alpha_{i} \in[0,1]} \operatorname{Var}(r)=\sum_{i=1}^{8} \alpha_{i}^{2} \sigma_{i}^{2}+2 \sum_{1 \leq i \leq j \leq 8} \alpha_{i} \alpha_{j} \sigma_{i j} \\
& \text { s.t. } \sum_{i=1}^{8} \alpha_{i} r_{i}=\bar{r} \\
& \sum_{i=1}^{8} \alpha_{i}=1 .
\end{aligned}
$$

The procedure will yield optimal shares of each type of technology. A set of optimal portfolios can be depicted on the return-risk plane (Fig. 3). The curve is the optimal portfolio frontier. Any point to the left is infeasible while any point to the right is considered sub-optimal.

The energy trilemma is partially addressed in the portfolio model because energy security is associated with "risk" and equity is associated with "return". The authors claim that in their framework, consumer welfare is maximized in terms of price stability, energy security, and clean-energy investment, implying that the third horn of the trilemma, sustainability, is also incorporated. However, clean energy only figures in the discussion because VRE sources are among the eight technologies considered. There is no explicit procedure by which lower carbon emissions can be targeted.

Unlike the application using Philippine data, the study of Stempien and Chan [15] makes categorical reference to the trilemma. Targeting "sustainability" is operationalized by adding another variable in the model: the expected return on emissions in terms of energy per unit of $\mathrm{CO}_{2}$, i.e., $\mathrm{kWh}$ per ton of $\mathrm{CO}_{2}$. Instead of having a twodimensional optimal portfolio frontier, the efficient plane is as depicted in Fig. 4. The three dimensions represent the constraints imposed by the trilemma under which the portfolio is optimized.

Neither the studies of Balanquit and Daway-Ducanes [13] and Stempien and Chan [15] provide a mechanism to choose among the options along the optimal portfolio frontier. This can be done by specifying a set of indifference curves-or planes in the multi-dimensional case. These are analogous to the aforementioned welfare function. The indifference curves (planes) are specified by determining the risk-return profile of the policymakers involved, which can also be accomplished through methods associated with MCDM (see Box 1).

The indifference curves should slope upward (Fig. 5). This indicates that in order for the investor to achieve the same level of utility, he must be compensated for accepting a greater level of risk with a higher expected rate of return. A higher indifference curve implies a higher level of utility. The choice of generation mix is where the indifference curve is tangent to the optimal portfolio frontier (point A in Fig. 5). In this framework, different policies will lead to various points in the risk-return plane. Policymakers should adopt the policy that generates the highest indifference curve or welfare.

\section{Box 1 Multi-criteria decision-making}

Multiple-criteria decision-making (MCDM) or multiple-criteria decision analysis (MCDA) falls under the discipline of operations research. MCDM is a set of methodologies that deal with multiple criteria in decision-making. The methodologies that are identified in the literature mostly differ in terms of assigning weights to the criteria involved. Among the methods are the aggregated indices randomization method (AIRM), analytic hierarchy process (AHP), analytic network process (ANP), balance beam process, basecriterion method (BCM), best-worst method (BWM), Brown-Gibson model, etc.

The AHP is applied in this study, the basic reference being Saaty [16]. By allowing the decision-maker to reveal his priorities, AHP streamlines a complex decision-making process. In a nutshell, a multifaceted process is reduced to a series of pairwise comparisons with the results being synthesized. AHP allows both subjective and objective aspects of a decision to be combined.

The AHP generates a weight for each evaluation criterion according to the decision-maker's pairwise comparisons of the criteria. The higher the weight, the more important is the corresponding criterion. 


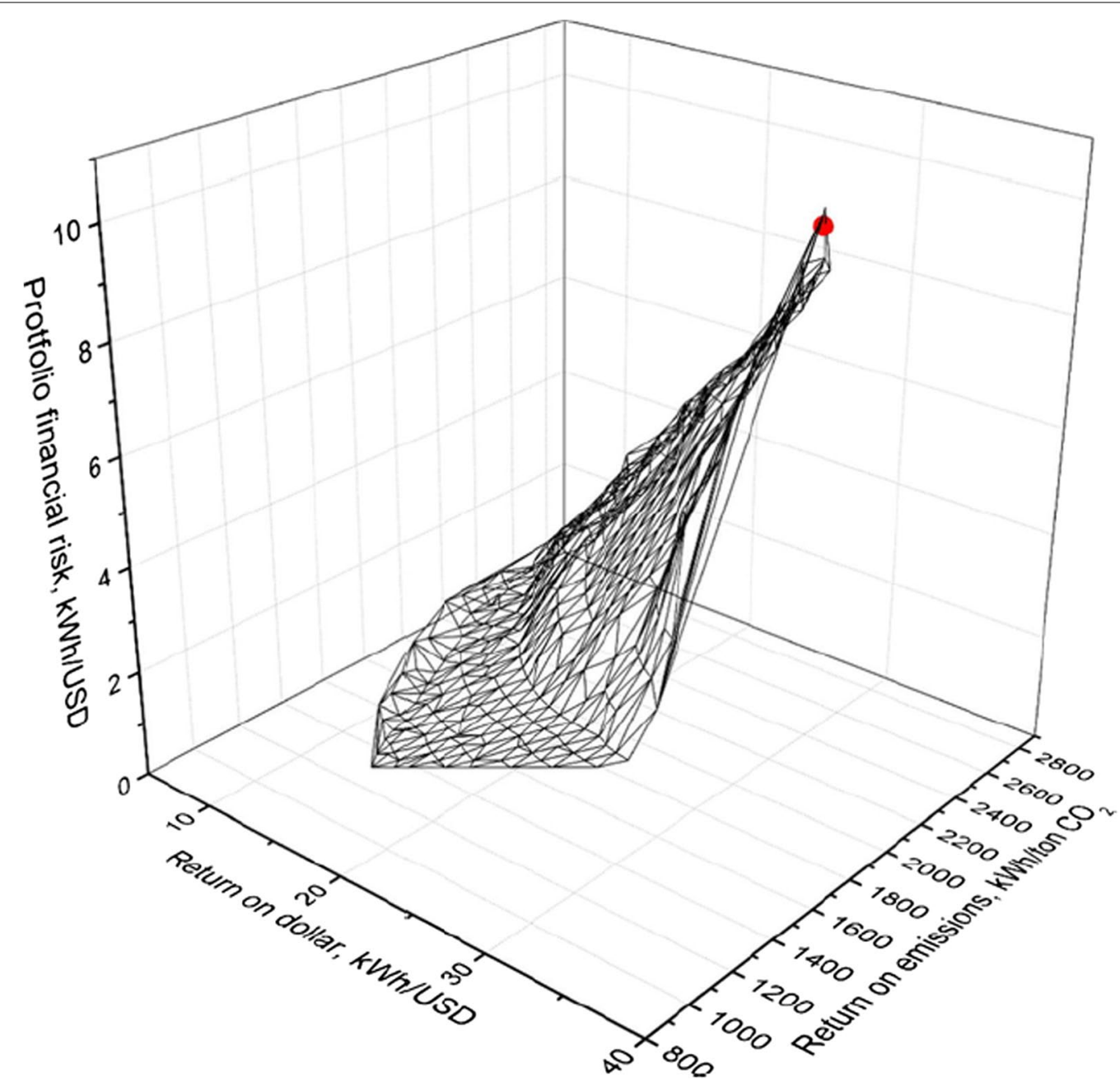

Fig. 4 Modified Markowitz theory of energy portfolio optimization. Source: Fig. 2 of [15]

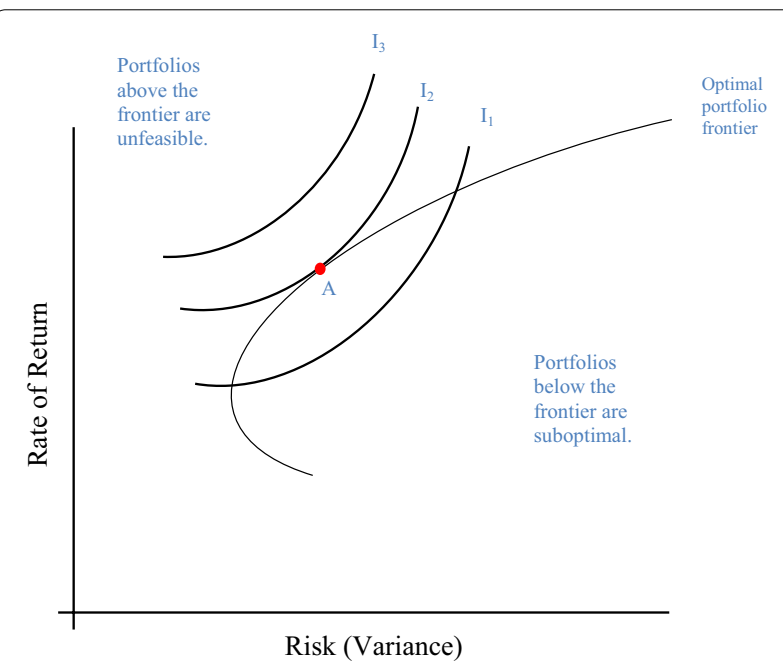

Fig. 5 Equilibrium (point A) between optimal portfolio frontier and the indifference curves of the hypothetical DOE secretary
To make pairwise comparisons, a scale of numbers is established in order to indicate how many times more important or dominant one criterion is over another. The table below presents the scale.

The fundamental scale of absolute numbers for AHP

Definition Preference scale

\begin{tabular}{lll}
\hline Equally preferred & 1 & Two criteria contribute
\end{tabular}

Equally to moderately 2

preferred

Moderately preferred $3 \quad$ Experience and judgment slightly favor one criterion over another

Moderately to strongly 4

preferred

Strongly preferred 5

Experience and judgment strongly favor one criterion over another 
The fundamental scale of absolute numbers for AHP

\begin{tabular}{lll}
\hline Definition & Preference scale \\
\hline $\begin{array}{l}\text { Strongly to very } \\
\text { strongly preferred }\end{array}$ & 6 & \\
\hline Very strongly preferred & 7 & $\begin{array}{l}\text { A criterion is favored very } \\
\text { strongly over another; its } \\
\text { dominance demonstrated } \\
\text { in practice }\end{array}$
\end{tabular}

Very strongly to extremely preferred

Extremely preferred

8

The evidence favoring one activity over another is of the highest possible order of affirmation

Source: Saaty [17], page 86

A more complicated process is the Stochastic Multicriteria Acceptability Analysis or SMAA (Lahdelma and Salminen [18]). This is a family of methods for aiding multi-criteria group decision-making in problems with uncertain, imprecise, or partially missing information. These methods are based on exploring the weight space in order to describe the preferences that make each alternative the most preferred one, or that would give a certain rank for a specific alternative. The main results of the analysis are rank acceptability indices, central weight vectors, and confidence factors for different alternatives. The rank acceptability indices describe the variety of different preferences resulting in a certain rank for an alternative, the central weight vectors represent the typical preferences favoring each alternative, and the confidence factors measure whether the criteria measurements are sufficiently accurate for making an informed decision."

SMAA was applied to the energy trilemma by Song et al. [19]. The different alternatives were evaluated based on three criteria which are the components of the trilemma. As an exercise, the authors used as alternatives the top ten countries based on the 2015 Energy Trilemma Index. Exact weights of the three criteria were not derived but these can be inferred from the reported rank acceptability indices.

"Lahdelma and Salminen [18], page 285.

\section{Methods and framework}

\section{Expanding the concept of energy security}

The IEA's website defines energy security as "the uninterrupted availability of energy sources at an affordable price. Energy security has many aspects: long-term energy security mainly deals with timely investments to supply energy in line with economic developments and environmental needs. On the other hand, short-term energy security focuses on the ability of the energy system to react promptly to sudden changes in the supplydemand balance."

Based on this rather broad definition, the concept of the trilemma is modified in this study. Energy governance seeks to promote energy security and one of the primary tasks is to manage the trade-off among its various components. Following the IEA's definition, these would be the major components to be considered: (1) adequate supply, (2) price, (3) environmental impact, and (4) ability to react promptly to sudden changes in the supplydemand balance. Hence, there is a "quadrilemma" among these components. Heretofore, however, the term "trilemma" is retained.

A simulation package is applied to generate values of these four variables over a selected time period under reasonable assumptions. Some of these assumptions reflect policy choices. The trade-offs and synergies among the components of the trilemma are embedded in the equations of the simulation model. The authors have access to PLEXOS and therefore the study is limited to power generation.

The main advantages of PLEXOS are the transparency of its methodology, flexibility in its application, and robustness of the results. Electricity demand can be scaled down to zonal and nodal levels, enabling the model to generate locational marginal prices. This is important given the archipelagic topography of the Philippines, which necessitates constructing an electric grid wherein marginal prices vary significantly. Meanwhile, PLEXOS can model physical elements of the system in a more detailed resolution. This implies that the bidding behavior of various plants can be modeled, allowing the idiosyncratic features of different energy sources to be incorporated. For example, the temporal nature of solar and wind power is readily defined, and specific features can vary on a regional and plant basis. Finally, unlike other commercial software, PLEXOS does not resort to heuristics. Instead, it takes advantage of the computational power of commercial LP Solvers to handle the problem of modeling and simulating the full Philippine power system even in the long term. The robustness of the results derives from the ability of PLEXOS to carry out sensitivity analysis, allowing users to simulate various scenarios. A consistency check of the results leads to confidence that algorithms are performed correctly. What should also be emphasized is that the framework

\footnotetext{
${ }_{5}^{5}$ https://www.iea.org/topics/energysecurity/ (Accessed 26 November 2019).
} 


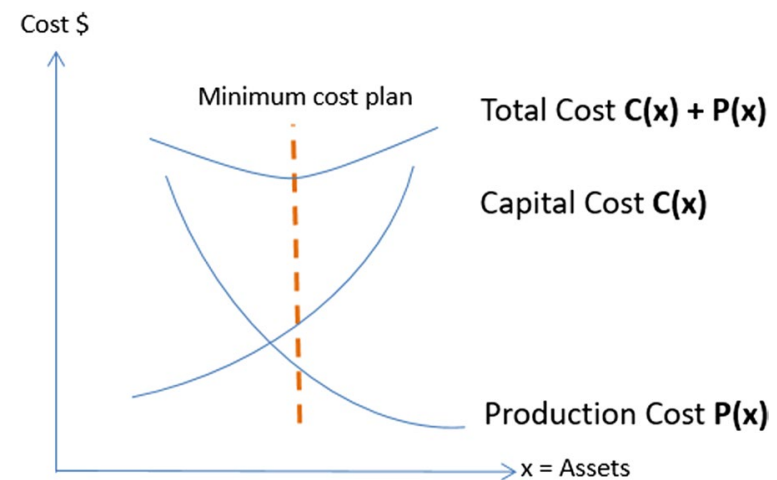

Fig. 6 Illustration of the objective of the LT Plan: minimize net present value of capital and production costs. Source: Energy Exemplar [20]

and methodology presented and applied in this study are invariant to the specific software and assumptions.

\section{Basic PLEXOS framework ${ }^{6}$}

The long-term (LT) phase plan of PLEXOS is discussed in this section to highlight the trade-offs and synergies
- Capital costs $\mathrm{C}(x)$, consisting of costs attributed to building new generator capacity and generator retirements. Generator build costs include the fixed amounts required to pay for capital and service debts.

- Production costs $\mathrm{P}(x)$, which include costs of operating the system using the existing plant line-up plus a basket of candidate builds. Also included in the formulation of production cost is the notional penalty of unserved energy.

Expansion candidates like variable renewable sources such as solar and wind are examples requiring relatively high capital costs and virtually minimal production costs. Liquid fuel resources such as oil-based generating units are expected to have high production costs. Adding carbon tax augments production costs of carbon-intensive generating resources, and hence, will prompt the simulator to look for a solution that moves away from these fossil fuel-based options, favoring renewable sources more.

The minimal formulation of the LT Plan is shown as follows:

Minimize:

$$
\begin{aligned}
& \sum_{(y)} \sum_{(g)} \mathrm{DF}_{y} \times\left(\text { BuildCost }_{g} \times \text { GenBuild }_{(g, y)}\right)+\sum_{(y)} D F_{y} \times\left[\text { FOMCharge }_{g} \times 1000 \times \text { PMAX }_{g}\left(\text { Units }_{g}+\sum_{i \leq y} \text { GenBuild }_{g, i}\right)\right] \\
& \quad+\sum_{t} D F_{t \in y} \times L_{t} \times\left[\operatorname{VoLL} \times \operatorname{USE}_{t}+\sum_{g}\left(\operatorname{SRMC}_{g} \times \text { GenLoad }_{g, t}\right)\right]
\end{aligned}
$$

among the components of the expanded trilemma: autarky (AT), affordability (P), Supply (S), and Sustainability as measured by carbon emissions $(\mathrm{C})$. The other components of PLEXOS are presented in the appendix. The LT phase seeks to solve the long-term generation capacity expansion problem by finding an optimal set of builds and simultaneously solving for the dispatch optimization problem from a central planner's perspective. In particular, the LT plan looks to identify what type of generator units to put in, where to put them in the system, and when to build it. This is further subjected to reliability constraints such as respecting capacity reserve requirements.

The general objective is to minimize net present value of capital and production costs of future generator build decisions and retirements (Fig. 6). Costs can be classified into two categories:

\footnotetext{
${ }^{6}$ PLEXOS is a high-performance simulation platform operationally used by energy market participants, system planners, investors, regulators, consultants, and analysts worldwide [20] The PLEXOS simulations are based on mathematical programming. The underlying structure of PLEXOS is described in this subsection and the appendix.
}

Subject to:

Energy balance constraint

$$
\sum_{(g)} \operatorname{GenLoad}_{(g, y)}+\mathrm{USE}_{t}=\operatorname{Demand}_{t} \forall_{t}
$$

Feasible energy dispatch

$$
\operatorname{GenLoad}_{(g, t)} \leq \operatorname{PMAX}\left(\text { Units }_{g}+\sum_{i \leq y} \text { GenBuild }_{g, i}\right)
$$

Feasible builds

$$
\sum_{i \leq y} \text { GenBuild }_{g, i} \leq \text { MaxUnitsBuilt }_{g, y}
$$

Integrality

$$
\text { GenBuild }_{(g, y)} \text { integer }
$$

Capacity adequacy 


$$
\sum_{(g)} \operatorname{PMAX}_{g}\left(\text { Units }_{g}+\sum_{i \leq y} \text { GenBuild }_{i}\right)+\text { CapShort }_{y} \geq \text { PeakLoad }_{y}+\operatorname{ReserveMargin}_{y} \forall_{y}
$$

\section{Definitions:}

\begin{tabular}{lll}
\hline Variable & Description & Type \\
\hline GenBuild $_{(g, y)}$ & $\begin{array}{l}\text { Number of generating units build in } \\
\text { year y for Generator } g\end{array}$ & Integer \\
GenLoad $_{(g, t)}$ & $\begin{array}{l}\text { Dispatch level of generating unit } g \text { in } \\
\text { period } t\end{array}$ & Continuous \\
USE $_{t}$ & Unserved energy in dispatch period $t$ & Continuous \\
CapShort $_{y}$ & Capacity shortage in year $y$ & Continuous
\end{tabular}

\begin{tabular}{|c|c|c|}
\hline Element & Description & Unit \\
\hline$D$ & $\begin{array}{l}\text { Discount rate. We then derive } \mathrm{DF}_{y}=1 / \\
(1+D)^{y} \text { which is the discount factor } \\
\text { applied to year, and } D F_{t} \text { which is the dis- } \\
\text { count factor applied to dispatch period } t\end{array}$ & \\
\hline$L_{t}$ & Duration of dispatch period $t$ & Hours \\
\hline BuildCost $_{g}$ & Overnight build cost of generator $g$ & $\$$ \\
\hline MaxUnitsBuilt $_{(g, y)}$ & $\begin{array}{l}\text { Maximum number of units of genera- } \\
\text { tor } g \text { allowed to be built by the end of } \\
\text { year } y\end{array}$ & \\
\hline PMAX $_{g}$ & $\begin{array}{l}\text { Maximum generating capacity of each } \\
\text { unit of generator } g\end{array}$ & MW \\
\hline Units $_{g}$ & $\begin{array}{l}\text { Number of installed generating units of } \\
\text { generator } g\end{array}$ & \\
\hline VoLL & Value of lost load (energy shortage price) & $\$ / M W h$ \\
\hline $\mathrm{SRMC}_{g}$ & $\begin{array}{l}\text { Short-run marginal cost of gen- } \\
\text { erator } g \text { which is composed of Heat } \\
\text { Rate } \times \text { Fuel Price + VO\&M Charge }\end{array}$ & $\$ / M W h$ \\
\hline FOMCharge $_{g}$ & $\begin{array}{l}\text { Fixed operations and maintenance charge } \\
\text { of generator } g\end{array}$ & $\$$ \\
\hline Load $_{t}$ & $\begin{array}{l}\text { Average power demand in dispatch } \\
\text { period } t\end{array}$ & MW \\
\hline PeakLoad ${ }_{y}^{7}$ & System peak power demand in year $y$ & MW \\
\hline ReserveMargin y & $\begin{array}{l}\text { Margin required over maximum power } \\
\text { demand in year } y\end{array}$ & MW \\
\hline CapShortPrice & Capacity shortage price & $\$ / M W$ \\
\hline
\end{tabular}

The formulation is illustrative only and is usually extended to include terms to handle candidate generators subject to inter-temporal constraints such as hydro

\footnotetext{
7 To determine energy demand and its peak, GDP/economic growth across the forecast horizon is obtained along with growth of energy demand. The historical relationship between these variables is then used to project energy demand quantities (GWh) and the peak load (MW). Implied growth rates of peak and energy demand are similar and are assumed in this exercise not to diverge across the horizon. To preserve temporal patterns of electricity consumption (whose seasonality is affected by variables like temperature), an hourly profile of a base year (most recent year) is used to serve as basis for the period-by-period load consumption of forecasted years.
}

energy limits, ramp-rate limitations, storage units like batteries, or contracts with minimum and maximum offtake requirements.

The following components of Energy Security are generated from PLEXOS: autarky (AT), affordability (P), supply (S), and sustainability (C). Autarky is defined as the share of energy from indigenous sources and is related to the ability to react promptly to sudden changes in the supply-demand balance. Affordability is equated to the price or cost of electricity. Meanwhile, the variable supply is proxied by the Capacity Reserve Margin $=($ Total generation capacity - peak load)/peak load.

Sustainability is a broad concept. As explained earlier, sustainable development requires that the principles of public policy be extended to the environomy-the union of the environment and the economy. This requires the inclusion of natural resource depletion and pollution in production and consumer-preference structures. ${ }^{8}$ This study simplifies the framework by using carbon emissions (C) as an indicator of sustainability. A more comprehensive set of indicators can be incorporated by expanding the welfare function.

\section{Applying the model}

Autarky (AT) is the annualized percentage of all indigenous generation $\left(\operatorname{GenLoad}_{(g, t)}\right)$ against the total generation of all sources. Indigenous sources include renewable generation such as wind, biomass, solar, and geothermal as well as resources fueled by domestic coal and gas. Recall that the model follows an economic dispatch algorithm. In order to satisfy the load at a minimum total cost, the set of generators with the lowest marginal costs are used first, with the marginal cost of the final generator needed to satisfy load requirements setting the system marginal price. System marginal prices are adjusted per location with considerations on cost of congestion and cost of losses to arrive at the locational marginal price. The affordability variable $(\mathrm{P})$ is the annual load weighted marginal price.

Meanwhile, the Supply variable (S) refers to the total built capacity of existing fleet plus additional generation fleet $\left(\right.$ GenBuild $_{(g, y)} \times$ PMAX $\left._{g}\right)$ to meet the peak demand and reserve margin of each year. The carbon emission variable $(\mathrm{C})$ refers to the carbon intensity. It is calculated

\footnotetext{
${ }^{8}$ The discussion on "sustainability" is based on Ravago and Roumasset [6] page 43 .
} 


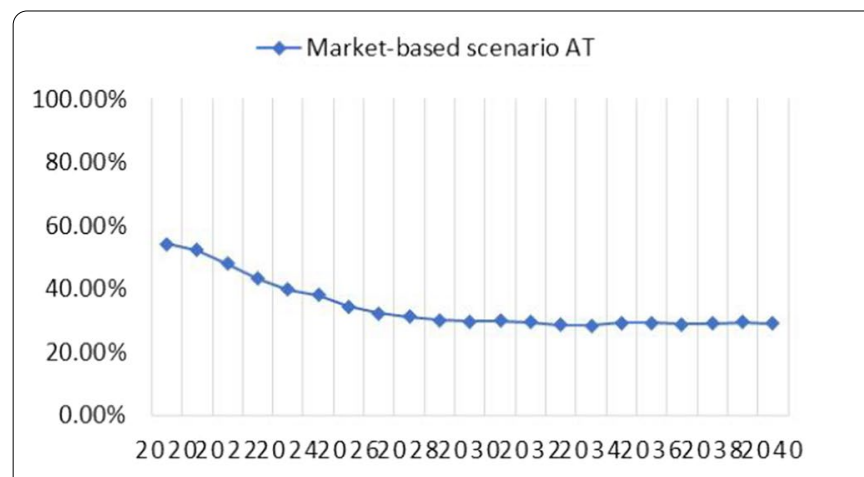

Panel A: Autarky Levels, market-based scenario

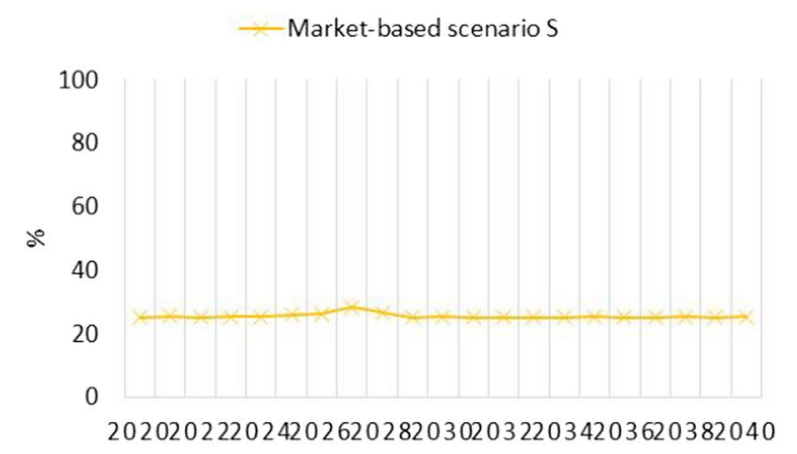

Panel C: Capacity Reserve Margin \%, market-based

Fig. 7 Market-based simulation results using PLEXOS

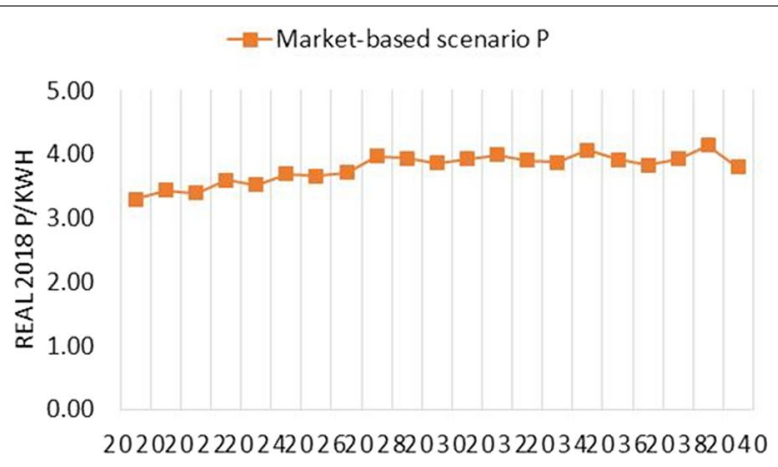

Panel B: Price in Php/kWh, market-based scenario

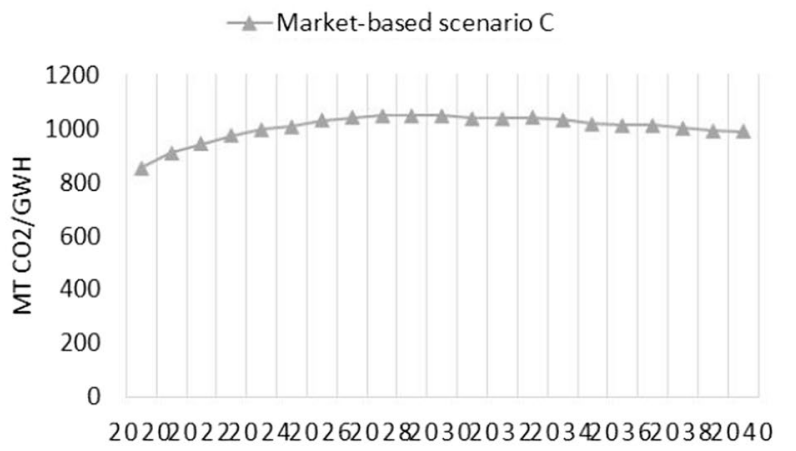

Panel D: Carbon Intensity $\mathrm{MTCO}_{2} / \mathrm{GWh}$, market-based by the summation of all emissions of carbon generating resources divided by the GWh generation in a year. Or simply, summation of $\operatorname{GenLoad}_{(g, t)} \times \mathrm{CO}_{2}$ emission factors divided by GenLoad $(g, t)$ of all resources in the system. All variables $\mathrm{AT}, \mathrm{P}, \mathrm{S}$, and $\mathrm{C}$ are further normalized to take a value of $0-1$.

In order to manage the trilemma, the variables will be combined in a welfare function, thus:

$$
W=\mathrm{AT}^{\alpha} \mathrm{P}^{\beta} \mathrm{S}^{\gamma} \mathrm{C}^{\delta} .
$$

The parameters $\alpha, \beta, \Upsilon, \delta$ are the weight of each factor in the welfare function and the most important objective is to maximize welfare, $W$. Let $W^{*}$ be the maximum welfare and by definition

$$
W^{*}=\mathrm{AT}^{\alpha *} \mathrm{P}^{\beta *} \mathrm{~S}^{\gamma *} \mathrm{C}^{\delta *} .
$$

Weights can be obtained through simulation-based optimization.

However, a more practical application is to obtain the weights of a hypothetical Secretary of the Department of Energy (DOE). His welfare function is $W^{H}=\mathrm{AT}^{\alpha H} \mathrm{P}^{\beta H} \mathrm{~S}^{\gamma h} \mathrm{C}^{\delta H}$, where the weights
$\alpha^{H}, \beta^{H}, \gamma^{H}, \delta^{H}$ can be obtained from the Analytical Hierarchy Process (or a similar procedure as described in Box 1). $W^{H}$ can then be used to evaluate policy options. As stated in the introduction, different policies will yield different values for the components of the trilemma, in this case AT, P, S, and C, thereby generating a set of values for $W$. The policy associated with the highest $W$ can then be selected and implemented. Similar to the argument made earlier, the framework is invariant to the specific methodology to obtain the weights.

It should be noted that in the actual simulation, the welfare function is defined as

$$
W=A T^{\alpha}\left(\frac{1}{\mathrm{P}}\right)^{\beta} \mathrm{S}^{\gamma}\left(\frac{1}{\mathrm{C}}\right)^{\delta} .
$$

A decline in both the price level and amount of carbon emissions increases welfare. Moreover, the four variables are normalized to a $[0,1]$ interval before $W$ is calculated.

For the portfolio model, instead of a welfare function, a utility function $U$ that depends on $r$ and $\sigma^{2}$ is defined, i.e., $U\left(r, \sigma^{2}\right)$. The appropriate weights for risk and return can also be determined through one of the MCDM 
Table 4 Preferences of two hypothetical DOE secretaries

\begin{tabular}{lllll}
\hline & $\mathbf{a}$ & $\mathrm{B}$ & $\Upsilon$ & $\boldsymbol{\delta}$ \\
\hline Secretary 1 & 0.42 & 0.12 & 0.28 & 0.18 \\
Secretary 2 & 0.25 & 0.25 & 0.25 & 0.25 \\
Secretary 3 & 0 & 1 & 0 & 0 \\
\hline
\end{tabular}

Source: Authors' calculations

procedures. If patterned after the welfare function, the utility function can be specified as: $U=(r)^{\alpha}\left(\sigma^{2}\right)^{\beta}$. Such an application is left for future study.

\section{Results and discussion}

Using PLEXOS, the power sector was forecast for the period 2020-2040 under a market-based scenario (Fig. 7). In this approach, the electricity market is assumed to unfold along a path where growing demand is automatically satisfied in the least cost manner. There is no mandated generation mix across the study period and no carbon tax is applied. Variable renewable energy costs are anticipated to continue along a significant downward trajectory. Meanwhile, domestic natural gas, as it depletes, gets replaced by the use of imported liquid natural gas (LNG).

Under the market-based scenario, coal remains to be a significant part of the mix as it is a cheap option for running on baseload function. The share of coal in the mix is anticipated to reach a peak of more than 70 percent in the first half of the study horizon. Renewable energy generation, on the other hand, is seen to rise to unprecedented levels starting in the second half of the period. In 2040, the share of solar generation is estimated to increase by more than 10 times its original share in 2020. Following this market-based scenario, autarky is expected to fall from a high level of 54 percent in 2020 to 30 percent in 2030. The drop is influenced by the increased dependence on imported fuel energy sources, namely coal, and the switch to imported LNG as local natural gas gets depleted.

Annual market price averages are projected to experience a slight increase from its initial price level by approximately $0.7 \mathrm{P} / \mathrm{kWh}$ (real 2018 terms) towards the period 2031-2040. The uplift is presumed to provide signals to encourage additional investment to support growing demand and reserve requirements. Capacity reserve margins remain stable at 25 percent throughout the horizon. Carbon intensity is anticipated to climb in the near term, starting from $854 \mathrm{tCO} 2 / \mathrm{GWh}$ in 2020 , reaching a peak of $1048 \mathrm{tCO} 2 / \mathrm{GWh}$ in 2030. This will slowly pull back to a level of $990 \mathrm{tCO} / \mathrm{GWh}$ in 2040. The rise of carbon intensity in the medium term is attributed to the increase in the share of thermal coal in the generation
Table 5 Value of W under market-based scenario

$W$ from Policy A (market-based results)

\begin{tabular}{ll}
\hline Secretary 1 & 0.0832 \\
Secretary 2 & 0.0912 \\
Secretary 3 & 0.6892 \\
\hline
\end{tabular}

Source: Authors' Calculations

mix. On the other hand, the slow decline of carbon intensity in the second half is a result of the proliferation of variable renewable resources.

Meanwhile, two energy experts were interviewed in order to obtain values for the parameters $\alpha, \beta, \Upsilon$, and $\delta$. They are identified as (hypothetical) Secretary 1 and Secretary 2. The Analytical Hierarchy Process was applied by presenting the four goals on a pairwise basis to each expert. There are six pairwise comparisons to be made. The basic process of AHP is described in Box 1 and the results are shown in Table 4.

Secretary 3 represents the optimal weights obtained from a simulation-based optimization procedure. These are the values $\alpha^{*}, \beta^{*}, \gamma^{*}, \delta^{*}$ described earlier. A corner solution is obtained meaning that all parameters are zero except for $\beta$ which is unity. This is not surprising since a policymaker who favors a market-based solution will definitely emphasize the least-cost alternative. Under the market-based scenario, the value of $W$ is calculated as follows (Table 5):

These are obtained by substituting the annual values (AT), affordability (P), supply (S), and sustainability (C) into Eq. (1) and getting the average of $W$ over the period 2020-2040.

To demonstrate the application of the framework in dealing with the trilemma, the policy of imposing a carbon tax is simulated. In this exercise, a carbon tax is imposed, equivalent to the social cost of carbon (SCC), which is estimated to be USD 47.2 Real 2018/MT $\mathrm{CO}_{2}$. The estimate is from the United States Environmental Protection Agency (US EPA). ${ }^{9}$ With an average discount rate of 3 percent, the social cost of carbon is USD 40.00 per metric ton of $\mathrm{CO}_{2}$ in 2018 using 2007 as a base year. This is converted to USD 47.2 to reflect current prices in 2018. Skeptics of climate change effects use a higher discount rate. At an average discount rate of 5 percent, the social cost of carbon falls to USD 12.00 per metric ton of $\mathrm{CO}_{2}$ in 2018. The debate on the appropriate level of carbon emissions and carbon tax is eschewed in this paper. ${ }^{10}$

\footnotetext{
${ }^{9}$ https://19january2017snapshot.epa.gov/sites/production/files/2016-12/ documents/sc_co2_tsd_august_2016.pdf (accessed 15 February 2020).

10 See for example Dietz and Stern [21].
} 


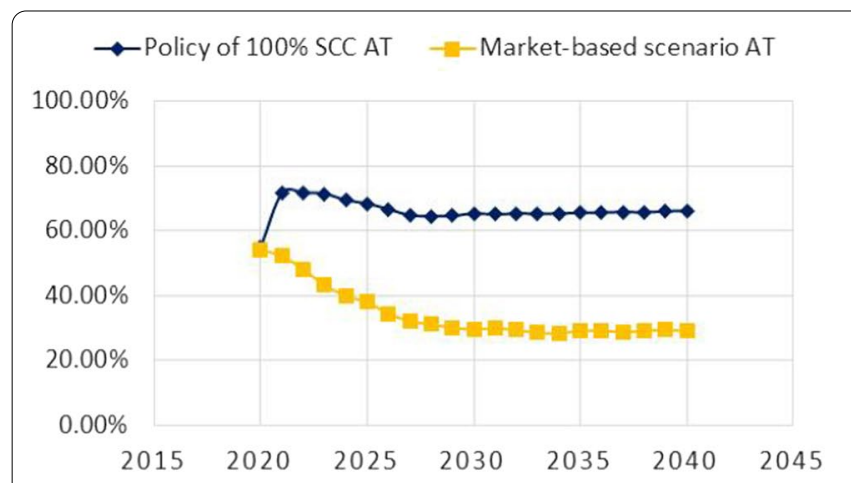

Panel A: Impact on Autarky

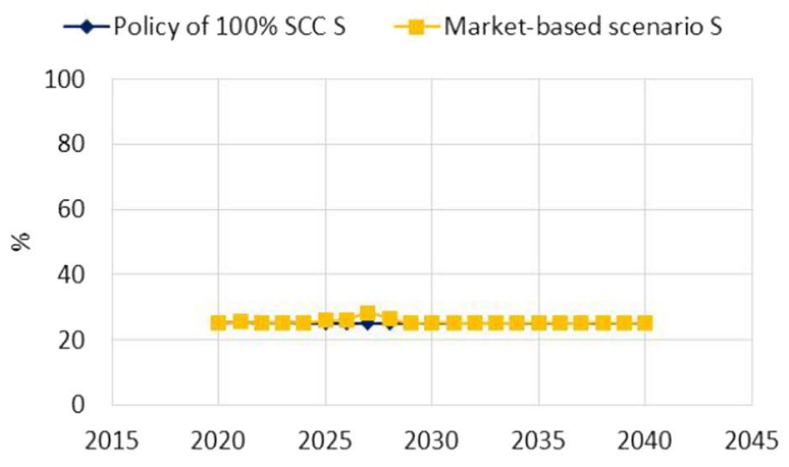

Panel C: Impact on Capacity Reserve Margin

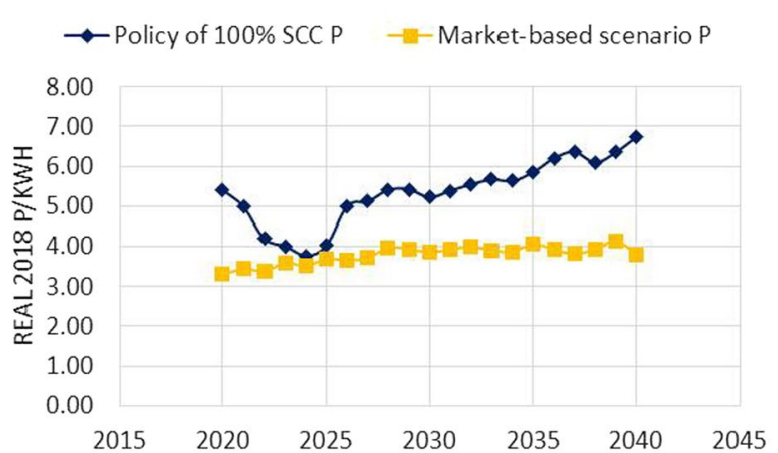

Panel B: Impact on Price

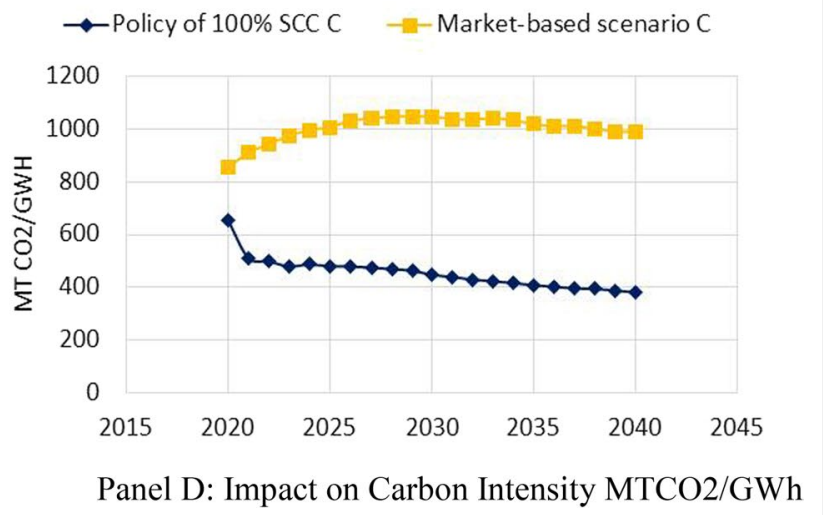

Fig. 8 Comparing market-based scenario with carbon tax scenario equal to $100 \%$ of social cost of carbon
Table 6 Comparing welfare before and after imposition of a carbon tax

\begin{tabular}{lll}
\hline & $\begin{array}{l}\text { W from Policy A (market- } \\
\text { based results) }\end{array}$ & $\begin{array}{l}\boldsymbol{w} \text { from policy b } \\
\text { (imposition of } \mathbf{a} \\
\text { carbon tax) }\end{array}$ \\
\hline Secretary 1 & 0.0832 & 0.2362 \\
Secretary 2 & 0.0912 & 0.2230 \\
Secretary 3 & 0.6892 & 0.2791 \\
\hline
\end{tabular}

Source: Authors' calculations

The carbon tax was incorporated in PLEXOS (see section on Basic PLEXOS Framework and Appendix) by adding to the short-run marginal cost (SRMC) of plants using coal, gas, and oil technologies. The appropriate emission factors were used.

The simulation results after imposing a carbon tax are shown in Fig. 8. The major trade-off involved in this policy exercise is a rise in the price of electricity accompanied by a decline in carbon emissions. In other words, enhanced sustainability is achieved at the cost of a decline in affordability. This will worsen equity if electricity demand of lower income classes has a higher price elasticity, which is the case in the Philippines (Dumagan and Abrigo [22]). The policy options can be evaluated by comparing the values of $W$ (Table 6).

Welfare improves under a government headed by Secretary 1 or Secretary 2. Welfare declines under an administration led by Secretary 3 .

It should be noted that the value of $W$ is higher under Secretary 3 for both policy regimes. Does this imply that Secretary 3 will be a more suitable head of the Department of Energy? Not at all. One can readily find a combination of values of the parameters and the variables that will generate a higher $W$. The parameters simply reflect the preferences of society. The welfare function is a mechanism to rank different policies given these parameters. What the results show is that both Secretary 1 and Secretary 2 will favor a carbon tax over a market-based scenario. Secretary 3 will not. 


\section{Conclusions}

The current application of the framework demonstrates its usefulness in avoiding a policy gridlock. Without the welfare function policymakers would grapple with the impact of the carbon tax. Would lower carbon emissions be an acceptable trade-off for higher cost of electricity and the accompanying rise in inequity? Comparing the value of the welfare function under the two scenarios would provide an objective basis for arriving at a decision. Progress can therefore be achieved even if the conflicts or trade-offs are not resolved. This is the essence of managing the trilemma.

Meanwhile, the reverse question can be investigated: given the parameters $\alpha, \beta, \Upsilon, \delta$, what would be the values of the components to maximize welfare? These can be designated as $\mathrm{AT}^{*}, \mathrm{P}^{*}, \mathrm{~S}^{*}, \mathrm{C}^{*}$. A time series for each variable can be generated. Policies can then be designed to target these values, with the full model taking into account the trade-offs and synergies.

Another logical extension of the model is to include economic variables such as per capita GDP and poverty incidence in the analysis. This can be readily accomplished by linking PLEXOS to a full-fledged macroeconomic model. The welfare function can then include relevant economic variables.

Policies that improve all components of the welfare function, while rare, can be designed. The Philippines should take advantage of the passage of Republic Act No. 11285 (An Act Institutionalizing Energy Efficiency and Conservation, Enhancing the Efficient Use of Energy, and Granting Incentives to Energy Efficiency and Conservation Projects) in 2019. Measures to improve energy efficiency will yield higher outputs or services from the same amount of resources. These measures include green building codes, minimum energy performance standards for equipment, and minimum standards for fuel efficiency, electric vehicles, and energy management systems industries. Improving energy efficiency can positively affect all components of the trilemma at the same time. This hypothesis can be verified by simulating the impact of measures to enhance energy efficiency.

La Viña et al. [10] point out that energy efficiency is part of the general strategy of demand-side management. This, in turn, is an element of an overall energy transition strategy called 'change of individual energy consumption behavior' (CIECB). Resolving the trilemma can be achieved by altering the individual energy consumption behavior which is characterized mainly the use and purchase of energy services and devices. By understanding factors that influence consumption behavior-such as income, education, age, geography, mindset-a CIECB governance approach could help in designing policies that generates energy efficiency through effective demand-side management.

\section{Appendix: PLEXOS Platform ${ }^{11}$}

PLEXOS is a commercial grade optimization-based software used to model electricity markets. The forecasting approach using PLEXOS is largely simulation-based, which is in contrast to other known practices where forecasts are done by regression. Its core simulation engine is centered on mixed-integer programming and the structure of the platform comprised interleaved simulation phases namely:

1. Long-term phase (LT Plan)

2. Projected assessment of system adequacy (PASA)

3. Medium-term schedule (MT Schedule)

4. Short-term schedule (ST Schedule)

The phases are solved in sequence and the output of one becomes the input to the succeeding simulation steps. The LT Plan was presented in the main text. PASA step looks to find the optimal timing of annual maintenance events of generating units. Outputs of LT and PASA steps are passed on to the MT and ST Schedules to further solve the more detailed dispatch optimization problem-the final solution of which contains parameters of interest such as the projected hourly dispatch schedule of individual generating unit and hourly system market prices.

\section{PASA phase}

The PASA simulation phase automatically schedules distributed maintenance events to equalize capacity reserves across peak periods (e.g., daily, weekly, monthly peak periods). Capacity reserve is the spare capacity over peak load in a region. Distributed maintenance events refer to outage periods typically required annually by generating plants to allow maintenance activities such as periodic maintenance, inspection of facilities, etc. Maintenance events are considered to occur in discrete periods and explicitly expressed to cover an expected number of hours and performed at a defined frequency in a year. This is in contrast to forced outage events where the number of times unplanned outages are drawn are implemented randomly.

\footnotetext{
${ }^{11}$ Excerpts from PLEXOS Wiki https://wiki.energyexemplar.com/ (Accessed November 30, 2019).
} 
The PASA phase is done after the LT phase when the annual future plant line-up is finalized. The distributed maintenance events are outputs of PASA and are passed down as input to the subsequent MT and ST simulation steps as optimal maintenance schedules. The optimal schedule of the PASA step is mainly based on capacity reserves only and not on production costs. This means maintenance timings handed down by PASA does not necessarily result in minimizing opportunity loss of an individual generator (due to lost revenue from the market).

\section{MT schedule}

MT schedule deals with the key problem in power system modeling which is to handle medium and long terms decisions in a computationally efficient way. In particular, this includes effectively addressing inter-temporal constraints present in energy-constrained generating units such as hydropower, storage units like battery, and contracts requiring fuel minimum/maximum off-takes by solving the economic dispatch optimization problem under a reduced chronology scenario.

To illustrate, take for example a forecast horizon spanning 20 years: The simulator is expected to simultaneously optimize decisions in the higher resolution level (in this case, hourly) while respecting medium-term constraints that span weeks for energy-constrained hydro generator or up to a year for a gas contract with minimum gas off-take. A simple approach would be to formulate $20 \times 8760 \mathrm{~h}=175,200$ dispatch intervals and solve it mathematically through one giant step. This simple approach, however, in reality, is computationally expensive and impossible to solve even with modern-day computers. To work around this, the MT Schedule finds an alternative solution over a reduced number of simulated periods by grouping together "similar" dispatch intervals and assigning them into blocks. Then, MT schedule optimizes decisions over this reduced chronology. The original medium-term constraints are then reduced into a set of equivalent short-term constraint targets and objectives that can be seamlessly integrated to the more detailed ST schedule that runs on full chronology. For example, given an energy-constrained hydropower plant with monthly limits-the MT schedule, because of its reduced number of chronological steps, will solve for an approximate hydro dispatch schedule based on the medium-term constraint. According to this approximate medium-term decisions, there is a set of shorter period target equivalents of the medium-term constraint that can be seamlessly passed on and enforced to the ST schedule-for instance, from monthly into daily energy targets. The ST schedule takes these daily targets as constraints added directly to the short-term formulation for its short-term dispatch policy.

Because MT schedule runs on a reduced chronology, it deals with constraints that span longer periods such as weeks, months, or even several years.

\section{Strategic bidding models}

Included in the MT schedule step are methods for strategic bidding such as Long Run Marginal Cost (LRMC) recovery and Residual Supply Index methodology. SRMC or short-run marginal costs refer to the variable costs of a generating unit's operation. LRMC refers to variable costs combined with the fixed costs covering fixed operation and maintenance and capital recovery fees to cover debt servicing and return to shareholders.

The PLEXOS LRMC cost recovery method is an automated price modification heuristic in which the price of generation from each Generator that belongs to a Company is modified to reflect the fixed cost burden of the Company as a whole. This price modification is dynamic, done iteratively, and designed to be consistent with the goal of recovering fixed costs across an annual time period.

Residual Supply Index (RSI) method is an empirical approach to modeling strategic bidding. It adopts a historical relationship (regression) between Price-cost Mark-up and certain system conditions and uses it to predict Bidcost Mark-up under future system conditions and applies the bid-cost mark-ups to the supply bids and runs the model to determine dispatch and market-clearing prices.

\section{ST schedule}

The ST schedule is a full chronological production cost simulation model used to emulate the dispatch and pricing of the real-time market clearing engine of the Wholesale Electricity Spot Market (WESM). The ST schedule solves both economic dispatch and unit commitment problems simultaneously.

In its core is the following economic dispatch and unit commitment formulation described as follows:

Minimize $F=\sum_{t=1}^{T} \sum_{i=1}^{N}\left[C_{i}\left(P_{\mathrm{Gi}}(t)\right)+S_{i}\left(u_{i}(t)\right)\right]$

Subject to: $\sum_{i=1}^{N} P_{G i}(t)=P_{D}^{\text {total }}+P_{\text {loss }}$ Power balance

$P_{\mathrm{Gi}}^{\min } \leq P_{\mathrm{Gi}} \leq P_{\mathrm{Gi}}^{\max }$ Gen. unit operating limit

$u_{i} \in[0,1]$ On or off

Other unit constraints Min up/downtime, ramp rate, etc.where:

- $C_{i}$ is fuel cost of gen unit $i$

- $S_{i}$ is start up or shut down cost of gen unit $i$

- $u_{i}$ is decision variable of start-up or shutdown of gen unit $i$

- $P_{\mathrm{Gi}}$ is generation output of gen unit $i$

- $P_{d}$ is total demand plus losses at time $t$

- $P_{\mathrm{Gi}}$ is generation output of gen unit $i$ 
- $P_{D}^{\text {total }}$ is total demand

- $P_{\text {loss }}$ is total transmission losses

Marginal prices and nodal prices:

The linear programming formulation described above refers to the primal problem which deals with physical quantities such as generation and demand. The formulation can be converted to a dual problem that primarily deals with economic values. The solution to the dual problem tells about the marginal price for energy which refers to the optimal value of the dual variable associated with the power balance constraint $\left(\sum P_{\mathrm{Gi}}(t)=P_{D}^{\text {total }}+P_{\text {loss }}\right)$. The marginal price represents the cost to system cost changes (in \$) for every one unit change in load (in MW).

The formula is as follows:

$$
\lambda=\delta C / \delta D
$$

where: $\lambda$ is the system lambda. $\delta C$ is the change in total system cost, $\$ . \delta D$ is the change in load, MW.

In a lossless transmission network and under no transmission constraints, the marginal prices across each electrical bus (represented by a trading node) are the same. Considering electrical network losses in the formulation results in separation of nodal prices. The same is true as network constraints causing congestion are introduced. The nodal price can be described as the system marginal price plus cost of losses and the cost of congestion.

$$
\lambda_{i}=\lambda+\alpha_{i}+\beta_{i}
$$

where: $\lambda_{i}$ is the nodal price. $\alpha_{i}$ is the node's cost of congestion. $\beta_{i}$ is the node's marginal loss charge.

\section{Acknowledgements}

The authors would like to acknowledge the financial support provided by the European Union and the Ateneo de Manila University School of Government through the Access to Sustainable Energy Program-Clean Energy Living Laboratories (ASEP-CELLS) project and the excellent research assistance of Joyce Marie P. Lagac. They would also like to express their appreciation to MERALCO PowerGen Corporation for allowing the use of the PLEXOS software. The usual disclaimer applies.

\section{Authors' contributions}

JTY is the main author and responsible for the framework and bulk of text in the paper. AJPG ran the simulations and prepared the write-up on the results and description of the PLEXOS software. CFH provided guidance on the structure of the simulations. All authors read and approved the final manuscript.

\section{Funding}

The study was conducted under the research component of the Access to Sustainable Energy Program-Clean Energy Living Laboratories (ASEP-CELLS) project which is funded by the European Union and managed by the Ateneo de Manila University School of Government. Neither the EU nor ASoG participated in the study.

\section{Availability of data and materials}

Data used in this study are available upon request, but can only be used to check the empirical results. Permission to use the software has to be obtained through Energy Exemplar, the custodian of the PLEXOS Market Simulation Software.

\section{Declarations}

\section{Ethics approval and consent to participate}

Not applicable.

\section{Consent for publication}

Not applicable.

\section{Competing interests}

The authors declare that they have no competing interests.

\section{Author details}

${ }^{1}$ Ateneo School of Government, Quezon City, Philippines. ${ }^{2}$ MERALCO PowerGen Corporation, Pasig City, Philippines.

Received: 22 January 2021 Accepted: 14 September 2021

Published online: 28 September 2021

\section{References}

1. World Bank Open Data (2021) https://data.worldbank.org/indicator/EG. ELC.ACCS.ZS. Accessed 21 January 2021

2. Index Mundi (2021) Electricity Consumption Per Capita. https://www. indexmundi.com/map/?v=81000. Accessed 23 January 2021

3. World Bank Open Data (2021) https://data.worldbank.org/indicator/NY. GDP.PCAP.KD. Accessed 31 July 2021

4. World Energy Council (2011) Policies for the Future, 2011 Assessment of Country Energy and Climate Policies av https://www.worldenergy.org/ assets/downloads/PUB_wec_2011_assessment_of_energy_and_clima te_policies_2011_WEC.pdf. Accessed 27 November 2019

5. World Energy Council (2019) World Energy Trilemma Index 2019. https:// www.worldenergy.org/assets/downloads/WETrilemma_2019_Full_ Report_v4_pages.pdf. Accessed 27 November 2019

6. Ravago MV, Roumasset JA (2018) The public economics of electricity policy with Philippine applications. In: Ravago MV, Roumasset JA, Danao RA (eds) Powering the Philippine economy: electricity economics and policy. University of the Philippines Press, Quezon City

7. Lazard (2019) Lazard's Levelized Cost of Energy Analysis_-Version 13.0. https://www.lazard.com/media/451086/lazards-levelized-cost-of-energyversion-130-vf.pdf. Accessed 11 August 2020

8. Barbier EB, Burgess JC (2019) Sustainable development goal indicators: analyzing trade-offs and complementarities. World Dev 1(22):295-305

9. World Energy Council (2020) World Energy Trilemma Index 2020, https:// www.worldenergy.org/assets/downloads/World_Energy_Trilemma_ Index_2020_-_REPORT.pdf (Accessed 21 January 2021)

10. La Viña AGM, Tan JM, Guanzon TIM, Caleda MJ, Ang L (2018) Navigating a trilemma: energy security, equity, and sustainability in the Philippines' low-carbon transition. Energy Res Soc Sci 35:37-47

11. Gunningham N (2013) Managing the energy trilemma: the case of Indonesia. Energy Policy 54:184-193

12. Lee, ND, Hurlbut, A, Dyreson, MI, McCan, I, Neri, EV, Reyes, NCR, Bagsik, J (2020) Ready for renewables: grid planning and competitive renewable energy zones (CREZ) in the Philippines. National Renewable Energy Laboratory and United States Agency for International Development, Golden

13. Balanquit R, Daway-Ducanes SL (2018) Optimal energy mix using portfolio theory. In: Ravago MV, Roumasset JA, Danao RA (eds) Powering the Philippine economy: electricity economics and policy. University of the Philippines Press, Quezon City

14. Markowitz H (1952) Portfolio selection. J Finance 7:77-91

15. Stempien JP, Chan SH (2017) Addressing energy trilemma via the modified Markowitz mean-variance portfolio optimization theory. Appl Energy 202:228-237

16. Saaty TL (1980) The analytic hierarchy process. McGraw Hill, New York

17. Saaty TL (2008) Decision making with the analytic hierarchy process. Int J Services Sci 1(1):83-98 
18. Lahdelma R, Salminen P (2010) Stochastic multicriteria acceptability analysis. In: Ehrgott M, Figueira JP, Greco S (eds) Trends in multiple criteria decision analysis. Springer Science + Business Media, LLC, New York

19. Song L, Fu Y, Zhou P, Lai K (2017) Measuring national energy performance via energy trilemma index: a stochastic multicriteria acceptability analysis. Energy Econ 66:313-319

20. Energy Exemplar (2015) PLEXOS 7.3 documentation. https://wiki.energ yexemplar.com/. Accessed 30 November 30, 2019

21. Dietz S, Stern N (2015) Endogenous growth, convexity of damages and climate risk: how Nordhaus' framework supports deep cuts in carbon emissions. Econ J 125:574-620
22. Dumagan, JC, Abrigo, MRM (2021) Rational choices and welfare changes in Philippine family energy demand: evidence from family income and expenditure surveys. Ateneo School of Government Working Paper 21-016. https://papers.ssrn.com/sol3/papers.cfm?abstract_id=3837066. Accessed 11 July 2021

\section{Publisher's Note}

Springer Nature remains neutral with regard to jurisdictional claims in published maps and institutional affiliations.
Ready to submit your research? Choose BMC and benefit from:

- fast, convenient online submission

- thorough peer review by experienced researchers in your field

- rapid publication on acceptance

- support for research data, including large and complex data types

- gold Open Access which fosters wider collaboration and increased citations

- maximum visibility for your research: over 100M website views per year

At BMC, research is always in progress.

Learn more biomedcentral.com/submissions 